\title{
Diamond and Related Nanomaterials for MEMS/NEMS Applications
}

\author{
Rakesh K. Joshi and Ashok Kumar \\ Department of Mechanical Engineering, University of South Florida, Tampa, FL 33620, USA \\ Correspondence should be addressed to Rakesh K. Joshi, joshi@eng.usf.edu
}

Received 28 July 2009; Accepted 28 July 2009

Copyright (c) 2009 R. K. Joshi and A. Kumar. This is an open access article distributed under the Creative Commons Attribution License, which permits unrestricted use, distribution, and reproduction in any medium, provided the original work is properly cited.

Diamond materials offer great potential for electronic and biomedical application. Properties like very high stiffness, thermal conductivity, optical transparency range, chemical stability and erosion resistance for the diamond based materials extend their applicability for several aspects of science and technology. Nanostructures of diamond materials have extraordinary multifunctional properties such as high thermal conductivity and a Youngs modulus value close to the one for single crystal diamond. Controlled nucleation and growth of nanocrystalline diamond along with their applicability for MEMS and NEMS structures are useful of future generation sensor platforms. Researchers around the world have been putting their efforts to achieve high precision in the direction of fabricating the devices based on diamond materials. Nanomanufacturing of such devices requires the controlled design of nanoscale diamond structures.

This issue covers various topics related to carbon-based nanomaterials. The first article by Hanada et al. reports deposition process of ultrananocrystalline diamond films. Authors have demonstrated application of pulse laser deposition for diamond growth. The second article (by Ohmagari et al.) in this issue is about atomic bonding configuration of ultrananocrystalline diamond (UNCD)/hydrogenated amorphous carbon (a-C: H) films, where authors have used near-edge X-ray absorption fine structure spectroscopy. The third article (by Teng et al.) is about structural transformation upon nitrogen doping of ultrananocrystalline diamond films. Tanaka et al. report high-resolution magnetic force microscopy using carbon nanotube probes in the fourth article of this issue. In the fifth article, Tung et al. report fabrication of carbon nanotubes STM tips by liquid catalyst-assisted microwave plasma-enhanced chemical vapor deposition. In a similar type of study, Sakamoto et al. (the sixth article) report fabrication of Pd-filled carbon nanotubes for SPM applications. In the seventh article (by Katzenmeyer et al.), the authors report Poly (hydridocarbyne) as highly processable insulating polymer precursor to micro/nanostructures and graphite conductors. In the concluding article, I. Chasiotis and K. N. Jonnalagadda report strength and fracture resistance of amorphous diamond-like carbon films for MEMS applications.

\section{Acknowledgments}

We thank all the contributing authors and reviewers for their help in putting together this special issue. The authors also thank the Editor-in-Chief of the Journal of Nanomaterials, Dr. Michael Z. Hu, for his support and encouragement. The authors highly appreciate Dr. Hu's contribution to the Journal of Nanomaterials.

Rakesh K. Joshi Ashok Kumar 

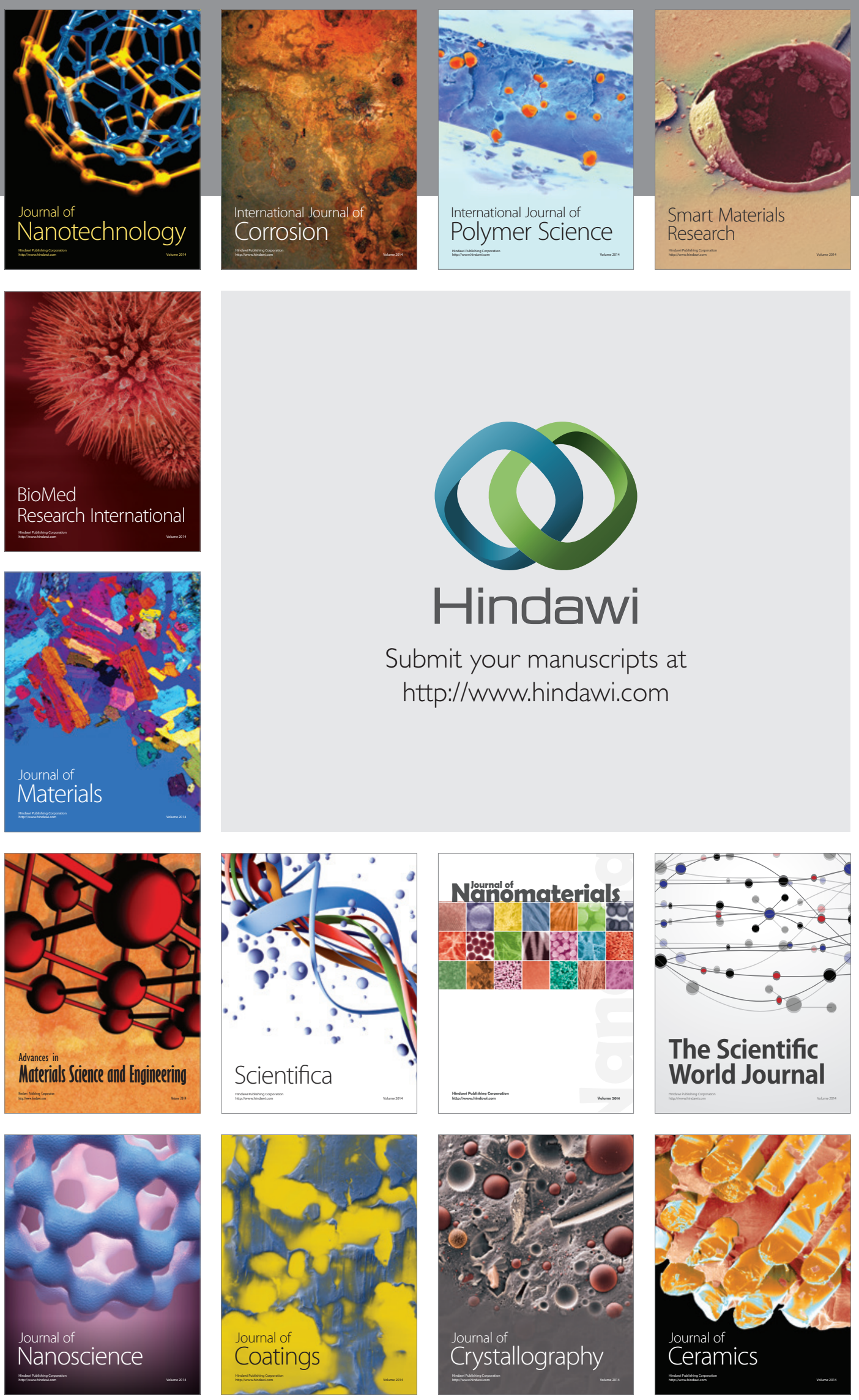

The Scientific World Journal

Submit your manuscripts at

http://www.hindawi.com

\section{World Journal}

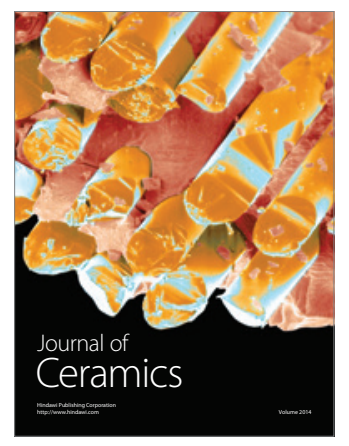

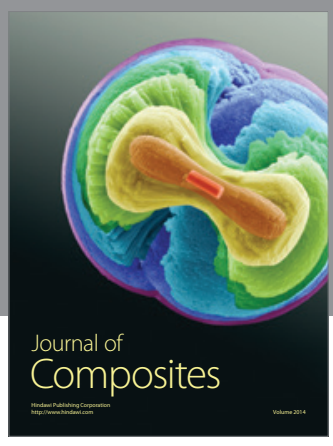
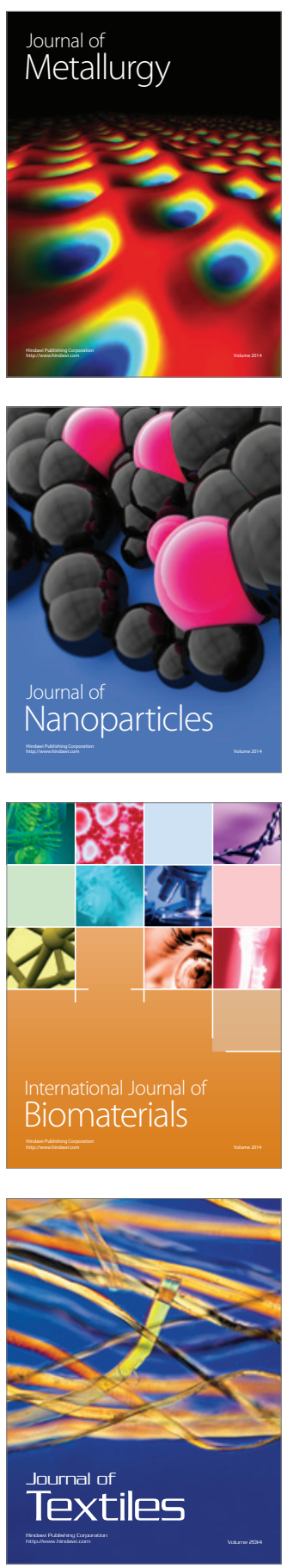